\title{
Preparation and Polymerization of $p$-Fluoromethylstyrene
}

\author{
Ryuzo Asami, Maung Gyi, ${ }^{*}$ Mikio TAKaki, and Toshiaki IkUta \\ Department of Synthetic Chemistry, Nagoya Institute of Technology, \\ Gokiso-cho, Showa-ku, Nagoya 466, Japan.
}

(Received October 11, 1977)

\begin{abstract}
Fluoromethylstyrene ( $p$-FMS) was prepared by means of a halogenhalogen interconversion reaction of $p$-chloromethylstyrene with potassium fluoride in the presence of a phase-transfer catalyst. As $p$-FMS is increasingly purified, it becomes less stable and undergoes the polycondensation of benzylic-type fluoromethyl groups. However, p-FMS was stable when traces of a basic solvent were added to it. Thus, in the presence of a small amount of acetonitrile, the homopolymerization of the monomer and the copolymerization with styrene (St) were carried out at $60^{\circ} \mathrm{C}$ using $\alpha, \alpha^{\prime}$-azobisisobutyronitrile as an initiator. The radical copolymerization of $p$-FMS with methyl methacrylate (MMA) takes place without any polycondensation even in the absence of a basic solvent, owing to the weak basicity of MMA itself. Monomer reactivity ratios of $p$-FMS $\left(M_{A}\right)$ with $\mathrm{St}$ and $\mathrm{MMA}\left(\mathrm{M}_{\mathrm{B}}\right)$ are $r_{\mathrm{A}}=1.3, r_{\mathrm{B}}=0.44$ and $r_{A}=0.84, r_{B}=0.30$, respectively. The polymers obtained were found to be stable both in solid state and in basic solvents.

KEY WORDS Halogen-Halogen Interconversion / p-Fluoromethylstyrene / Basic Solvent / Radical Polymerization / Monomer Reactivity Ratio /
\end{abstract}

Although the chloromethylation of polystyrene has been performed mostly for the preparation of polymers having benzylic-type chloromethyl groups, recently the homo- and copolymerization $^{1,2}$ of chloromethylstyrene (CMS), which is now commercially available, were recommended to obtain the well-defined polymers. However, no reports have been published on the synthesis of polymers containing fluoromethyl groups by either the fluoromethylation of polystyrene or the polymerization of fluoromethylstyrene monomers.

In the recent literature, ${ }^{3}$ it has been shown that the chlorine of organic chlorides can be successfully replaced by fluorine in the presence of a phase-transfer catalyst. As the application of this method, we have carried out the halogenhalogen interconversion reaction of $p$-CMS with potassium fluoride in the presence of the catalyst.

* Present Address: Plastic Factory No. 2, Pharmaceutical Industries Corporation, Rangoon, Burma.
The present study deals with the preparation of $p$-FMS and its radical homopolymerization and copolymerization with vinyl monomers.

\section{EXPERIMENTAL}

\section{Materials}

Commercial potassium fluoride (KF) and commercial cetyltrimethylammonium bromide (CTMAB), a phase-transfer catalyst, were used without further purification. $p$-Chloromethylstyrene ( $p$-CMS) was prepared by the method described in another paper. $^{2}$ Methyl methacrylate (MMA), styrene (St), and $\alpha, \alpha^{\prime}$-azobisisobutyronitrile (AIBN) used in the copolymerizations were purified by the usual methods.

\section{Procedures}

Preparation of $p$-FMS from $p$-CMS. In a $500-\mathrm{m} l$ three-necked round-bottom flask were placed $60 \mathrm{~g}$ of $p$-CMS, $186 \mathrm{~g}$ of $\mathrm{KF}, 11.8 \mathrm{~g}$ of CTMAB, which is essential to this kind of 
reaction, ${ }^{3-5} 70 \mathrm{~m} l$ of toluene, $105 \mathrm{ml}$ of water, and a small amount of $p$-tert-butylcatechol. The mixture was stirred vigorously and heated in an oil bath of $120^{\circ} \mathrm{C}$ (temp of the mixture, $113^{\circ} \mathrm{C}$ ). In order to avoid prolonged heating of the reaction mixture, the extent of conversion of $p$-CMS was followed by measuring the residual $p$-CMS with gas chromatography (GC). When the extent of conversion reached a maximum, stirring was stopped and the hot mixture was allowed to separate into two layers. The lower aqueous layer was removed from the flask by a siphon while the layer was hot. The yield of $p$-FMS was found to increase to some extent when the aqueous solution was renewed in the course of the reaction. The organic compounds contained in the aqueous layer removed were extracted with ether and added to the organic layer. The organic layer was washed several times with water containing sodium sulphate, dried over sodium sulphate, and poured into methanol to separate the polymer formed during the interconversion reaction. After the solvent was evaporated and small amounts of hexamethylphosphoric triamide (HMPA) and p-tertbutylcatechol were added to its residue, the mixture was distilled under a reduced pressure (7.0 mmHg), giving $26 \mathrm{~g}$ of $p$-FMS as a fraction of $45.5^{\circ} \mathrm{C} . \quad{n_{\mathrm{D}}}^{20}=1.5347 . \quad d_{4}{ }^{20}=1.22$. Fluorine content was $13.7 \%$ (calcd, 13.95\%). In the receiving flask, a small amount of potassium carbonate was placed beforehand. HMPA and potassium carbonate were added in order to stabilize the fluoromethyl groups, as will be described later.

Polymerization. The homopolymerization of $p$-FMS and its copolymerization with $\mathrm{St}$ were carried out as described in another paper, ${ }^{2}$ with the exception that acetonitrile was added to $p$-FMS before the polymerization as a stabilizer.

\section{Analysis and Measurements}

The composition of the copolymers was determined mostly by NMR analysis. Some of these results were checked by elemental analyses of those copolymers and the coincidence was satisfactory. NMR spectra were recorded using a Hitachi R-20B (60 MHz) spectrometer. IR spectra were obtained on a spectrometer of Model IRA-1 of JASCO. Gel-permeation chromatograms (GPC) were measured in tetrahydrofuran (THF) using a Toyo Soda HLC802UR with two GHM columns.

\section{RESULTS AND DISCUSSION}

\section{Preparation of p-FMS}

Table I summarizes the results of the halogen-

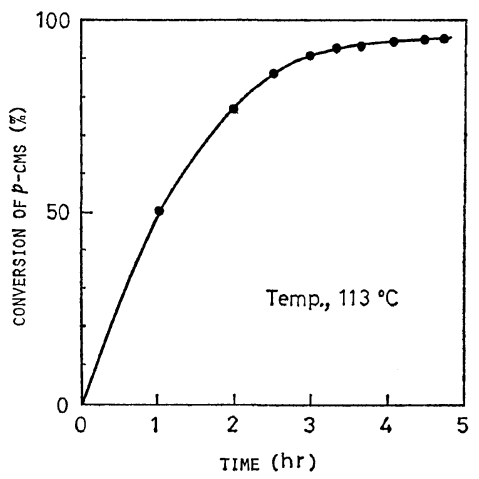

Figure 1. Time-conversion curve of $p$-CMS in the halogen-halogen interconversion of $p$-CMS with potassium fluoride. The extent of conversion of $p$-CMS was obtained from residual $p$-CMS determined by GC.

Table I. Halogen-halogen interconversion reaction of $p$-CMS with potassium fluoride

\begin{tabular}{|c|c|c|c|c|c|c|c|c|c|c|}
\hline \multicolumn{2}{|c|}{$p$-CMS } & & \multicolumn{2}{|c|}{$\mathrm{KF}$} & \multicolumn{2}{|c|}{$\mathrm{CTMAB}^{\mathrm{b}}$} & \multirow{2}{*}{$\begin{array}{l}\text { Toluene, } \\
\mathrm{m} l\end{array}$} & \multirow{2}{*}{$\begin{array}{l}\mathrm{H} \mathrm{H}_{2} \mathrm{O} \\
\mathrm{m} l\end{array}$} & \multirow{2}{*}{$\underset{\mathrm{hr}}{\mathrm{Time}}$} & \multirow{2}{*}{$\begin{array}{l}p \text {-FMSe } \\
\text { yield, } \%\end{array}$} \\
\hline g & $\mathrm{mol}$ & & $\mathrm{g}$ & $\mathrm{mol}$ & g & $\mathrm{mol}$ & & & & \\
\hline 34 & 0.223 & & 106 & 1.82 & 6.7 & 0.018 & 40 & 60 & 4 & 18 \\
\hline 60 & 0.393 & & 186 & 3.20 & 11.8 & 0.032 & 70 & 105 & 6 & 26 \\
\hline 60 & 0.393 & $\left\{\begin{array}{l}1 \text { st } \\
2 \text { nd }\end{array}\right.$ & $\begin{array}{l}170 \\
170\end{array}$ & $\begin{array}{l}2.93 \\
2.93\end{array}$ & $\begin{array}{r}11.0 \\
6.5\end{array}$ & $\begin{array}{l}0.030 \\
0.018\end{array}$ & $\underline{70}$ & $\begin{array}{r}100 \\
70\end{array}$ & $\left.\begin{array}{l}1 \\
4\end{array}\right\}^{\mathrm{d}}$ & 39 \\
\hline
\end{tabular}

a Reaction temp, $113^{\circ} \mathrm{C}$.

b CTMAB, cetyltrimethylammonium bromide.

e bp, $62.5^{\circ} \mathrm{C} / 9.5 \mathrm{mmHg} ; 45.5^{\circ} \mathrm{C} / 7.0 \mathrm{mmHg}$.

a The aqueous solution was renewed in the course of the reaction. 


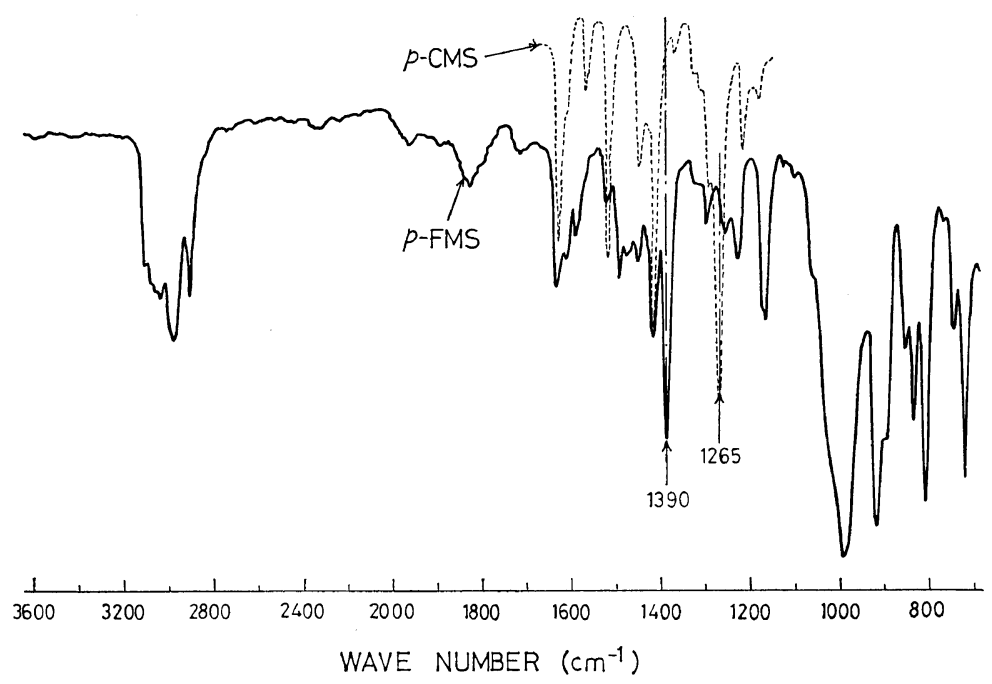

Figure 2. Infrared spectra of $p$-FMS $(-)$ and $p$-CMS (--).

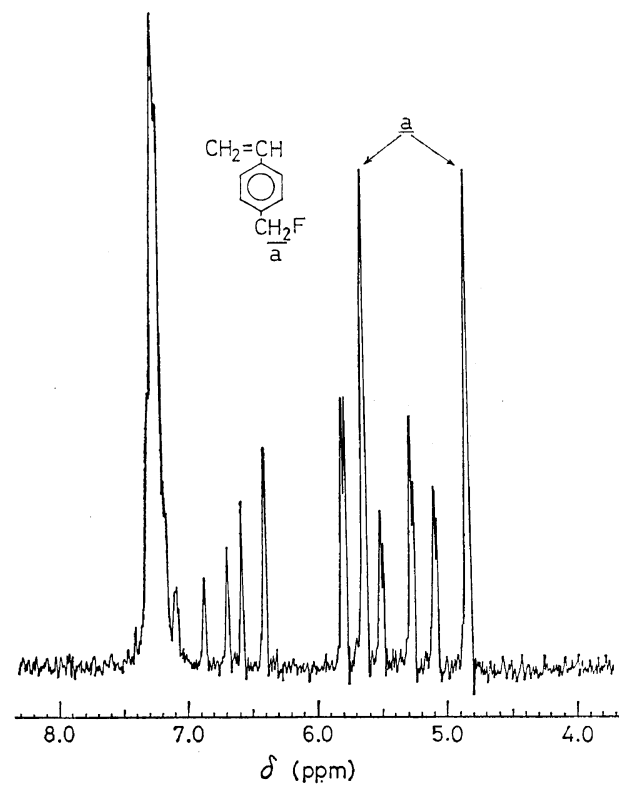

Figure 3. NMR spectrum of p-FMS (ca. 5\% in $\left.\mathrm{CCl}_{4}\right)$.

halogen interconversion reaction of $p$-CMS with $\mathrm{KF}$ in the presence of CTMAB as a catalyst. Figure 1 shows the relation between reaction time and the extent of conversion of $p$-CMS which was obtained from the residual $p$-CMS determined by GC. Although the extent of conversion of $p$-CMS is above $90 \%$, the yield of $p$-FMS is appreciably lower. The low yield of isolated $p$-FMS may be due to the high reaction temperature, which causes the thermal polymerization of vinyl groups in the course of the interconversion reaction and is indispensable to this reaction. ${ }^{3}$ The favorable reaction time is found to be $4-6 \mathrm{hr}$ from Table $I$ and Figure 1 under these reaction conditions.

The GC of $p$-FMS prepared by the above method showed a single peak. In the IR spectrum of $p$-FMS (Figure 2), the $-\mathrm{CH}_{2} \mathrm{Cl}$ peak at $1265 \mathrm{~cm}^{-1}$ gave place to the $-\mathrm{CH}_{2} \mathrm{~F}$ peak at $1390 \mathrm{~cm}^{-1}$. Figure 3 shows the NMR spectrum $\left(\mathrm{CCl}_{4}\right)$ of $p$-FMS, where the phenyl, vinyl, and fluoromethyl proton signals appeared at $\delta=7.27$, $5.08-6.88$, and 5.22 (doublet, $J_{\mathrm{H}-\mathrm{F}}=47 \mathrm{~Hz}$ ), respectively. The ratio of the peak areas was $4.0: 4.0: 3.01: 2.0$.

It was found that $p$-FMS is an extraordinarily labile monomer and less stable as it is more purified. In most cases, when no stabilizer was used, the distillate of $p$-FMS polymerized almost explosively, with evolution of heat and fumes during distillation. Even if such polymerization did not take place during the distillation, the distilled $p$-FMS kept at room temperature suddenly polymerized after an induction period of a few hours. This polymerization reaction was found to be a polycondensation of FriedelCrafts-type which was reported in an early ref 
6 and was confirmed by our recent study ${ }^{7}$ on the non-catalyzed polymerization of benzyl fluoride and its related reactions. However, we found that the fluoromethyl group of $p$-FMS was made stable for a long time in the presence of a small amount of HMPA, THF, acetonitrile, or basic inorganic compounds such as potassium carbonate, whereas benzene, nitrobenzene, and nitromethane were not effective. This suggests that the basicity of these substances was responsible for the stabilization of $p$-FMS. Now it is understood that the strong basicity of CTMAB stabilized the produced $p$-FMS during the halogen-halogen interconversion reaction even at a high temperature of $113^{\circ} \mathrm{C}$. Accordingly, we would like to suggest that it is important for the storage of this monomer to add a small amount of a basic solvent or potassium carbonate to the monomer.

Homopolymerization of $p-F M S$

As expected from the preceding discussion, no sooner had the ampoule containing pure $p$ FMS and 1 wt $\%$ of AIBN been immersed in a thermostat kept at $60^{\circ} \mathrm{C}$ than $p$-FMS polymerized explosively and the polymer filled up the ampoule. This polymer is insoluble in THF, benzene, and $N, N$-dimethylformamide. The insolubility of the polymer is obviously due to the gelation reaction, where both the polycondensation of benzylic-type fluoromethyl groups and the addition polymerization of vinyl groups take place simultaneously. However, as described above, only the vinyl polymerization of $p$-FMS took place at a moderate rate, even at $60^{\circ} \mathrm{C}$, when a small amount of basic solvent was added to $p$-FMS as a stabilizer. In this study, acetonitrile was used as the basic solvent by reason of the fact that the chain-transfer constant of styryl radical to the solvent is relatively low $\left(C_{s}=2.45 \times 10^{-5}\right.$ at $\left.60^{\circ} \mathrm{C}^{8}\right)$. The results of the homopolymerization of $p$-FMS are given in Table II. The polymer thus obtained was soluble in solvents which dissolve polystyrene, such as THF, benzene, $N, N$-dimethylformamide, etc. In the NMR spectrum of poly( $p$-fluoromethylstyrene), PFMS, two broad peaks at $\delta=6.97$ and $6.48 \mathrm{ppm}$ (phenyl protons), a doublet peak at $5.21 \mathrm{ppm}\left(J_{\mathrm{H}-\mathrm{F}}=47 \mathrm{~Hz}\right)$, (fluoromethylene protons), and a broad peak at $\delta=1.5 \mathrm{ppm}$ (methylene and methyn protons) appeared and assigned (Figure 4). The ratio of the peak areas was $4.0: 2.0: 3.1$, suggesting that only the addition polymerization of the vinyl group of $p$-FMS took place.

When PFMS was kept in its solid state, the pendant fluoromethyl groups of PFMS were stable, i.e., the IR spectrum of the polymer did not change at room temperature even after three months. Also, the polymer was stable in a

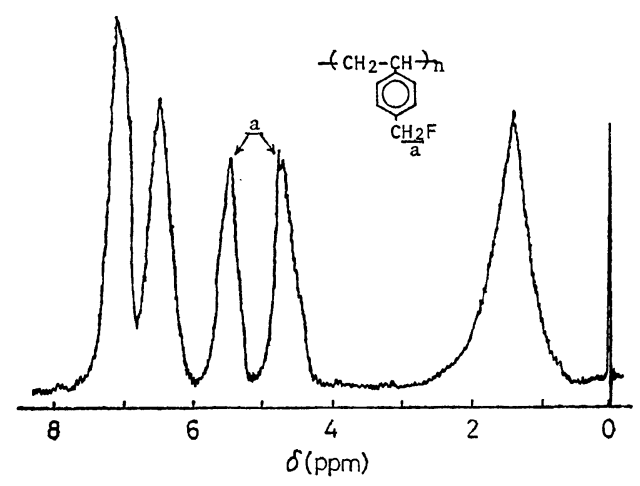

Figure 4. NMR spectrum of poly(p-flouoromethylstyrene) (ca. $5 \%$ in $\mathrm{CCl}_{4}-\mathrm{CDCl}_{3}$ mixture).

Table II. Homopolymerization of $p$-FMS

\begin{tabular}{ccccccc}
\hline $\begin{array}{c}p \text {-FMS, } \\
\mathrm{g}\end{array}$ & $\begin{array}{c}\text { AIBN, } \\
\mathrm{mg}\end{array}$ & $\begin{array}{c}\text { Benzene, } \\
\mathrm{m} l\end{array}$ & $\begin{array}{c}\text { Acetonitrile, } \\
\mathrm{m} l\end{array}$ & $\begin{array}{c}\text { Time, } \\
\mathrm{hr}\end{array}$ & \multicolumn{2}{c}{ Polymer } \\
\hline 2.0 & 21 & 2.0 & 0 & 1 & $95^{\mathrm{c}}$ & - \\
0.5 & 11 & 0.5 & 0.2 & 20 & 51 & 6.4 \\
3.2 & 38 & 2.0 & 1.0 & 23 & 83 & $3.1^{\mathrm{d}}$ \\
\hline
\end{tabular}

a Temp, $60^{\circ} \mathrm{C}$.

b Based on GPC, calibrated with standard polystyrene.

- Gelation.

a Fluorine content: found, 13.6\%; calcd, $13.95 \%$. 
Polymerization of $p$-Fluoromethylstyrene

Table III. Radical copolymerization of $p$-FMS with Sta

\begin{tabular}{|c|c|c|c|c|c|c|c|}
\hline No. & $\begin{array}{l}p \text {-FMS } \\
\left(\mathbf{M}_{\mathrm{A}}\right) \\
\text { mmol }\end{array}$ & $\begin{array}{c}\mathrm{St} \\
\left(\mathrm{M}_{\mathrm{B}}\right) \\
\mathrm{mmol}\end{array}$ & $\begin{array}{l}\text { Mol fract } \\
\text { of } M_{A} \\
\text { in feed }\end{array}$ & $\begin{array}{c}\text { Time, } \\
\text { min }\end{array}$ & $\begin{array}{c}\text { Conversion, } \\
\%\end{array}$ & $M_{n}^{\mathrm{b}} \times 10^{-5}$ & $\begin{array}{l}\text { Mol fract } \\
\text { of } \mathbf{M}_{A} \text { in } \\
\text { copolymerization }\end{array}$ \\
\hline S- 1 & 2.57 & 16.3 & 0.136 & 45 & 6.4 & - & 0.26 \\
\hline S- 2 & 3.93 & 12.9 & 0.233 & 45 & 4.8 & 1.4 & 0.34 \\
\hline S- 3 & 5.99 & 12.1 & 0.331 & 40 & 6.8 & - & 0.46 \\
\hline S- 4 & 7.93 & 11.1 & 0.417 & 50 & 5.6 & 2.0 & 0.53 \\
\hline S- 5 & 9.22 & 10.4 & 0.470 & 40 & 7.6 & - & 0.58 \\
\hline S- 6 & 10.7 & 7.52 & 0.587 & 50 & 8.5 & - & 0.68 \\
\hline S- 7 & 11.9 & 5.30 & 0.692 & 40 & 6.8 & 2.0 & 0.75 \\
\hline S- 8 & 13.1 & 5.07 & 0.721 & 35 & 7.7 & - & 0.78 \\
\hline S- 9 & 12.0 & 3.28 & 0.785 & 50 & 7.6 & 1.4 & 0.84 \\
\hline S-10 & 13.2 & 1.74 & 0.883 & 40 & 11.0 & - & 0.93 \\
\hline
\end{tabular}

a Temp, $60^{\circ} \mathrm{C}$; initiator, AIBN $(0.5 \mathrm{wt} \%)$; acetonitrile, $5 \mathrm{wt} \%$ of an amount of $p$-FMS.

b Based on GPC, calibrated with standard polystyrene.

basic solvent or a hydrocarbon solvent containing a small amount of a basic solvent. However, when the polymer was dissolved in purified benzene, the polymer solution gradually became turbid and then turned into a jellylike mass after a day or less.

\section{Copolymerization with St or MMA}

The radical copolymerization of $p$-FMS $\left(\mathrm{M}_{\mathbf{A}}\right)$ with St $\left(\mathbf{M}_{\mathbf{B}}\right)$ was performed in the presence of a small amount of acetonitrile. The results are given in Table III. The Fineman-Ross plots gave a straight line and the reactivity ratios, $r_{\mathrm{A}}$ and $r_{\mathrm{B}}$, were estimated to be 1.3 and 0.44 , respectively. By the use of these reactivity ratios, the composition curve for the copolymerization was drawn as shown in Figure 5. The experimental plots are in close agreement with the calculated composition curve.

In the radical copolymerization of $p$-FMS with MMA, only the vinyl copolymerization took place without any polycondensation of the fluoromethyl group, even in the absence of any basic solvent. This suggests that a week basicity of MMA itself is enough to supress the polycondensation. Thus the copolymerization of $p$-FMS $\left(\mathrm{M}_{\mathrm{A}}\right)$ with MMA $\left(\mathrm{M}_{\mathrm{B}}\right)$ was carried out without the addition of a basic solvent. The copolymerization results are summarized in Table IV. The reactivity ratios estimated from the FinemanRoss plots are $r_{\mathrm{A}}=0.84$ and $r_{\mathrm{B}}=0.30$. The composition curve obtained from these values agrees with the experimental plots (Figure 5). It is also noted that the composition curve for the

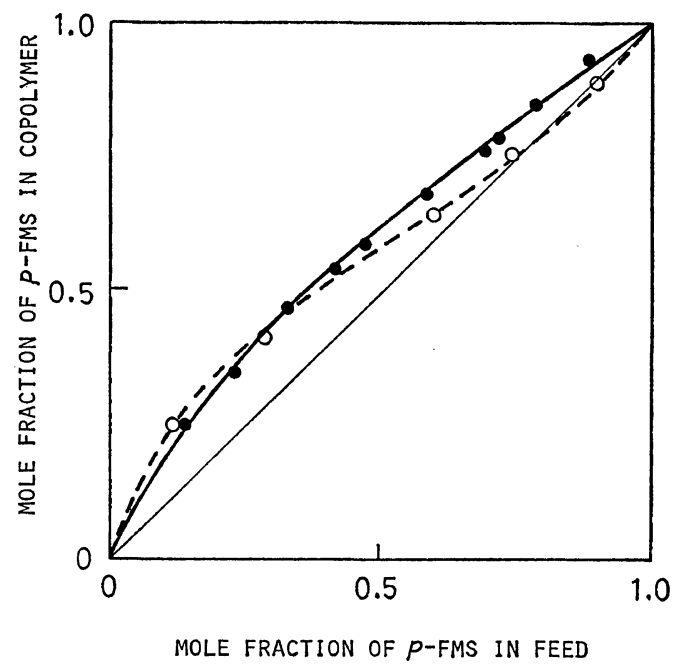

Figure 5. Composition curves for the copolymerization of $p$-FMS $\left(\mathrm{M}_{\mathrm{A}}\right)$ with $\mathrm{St}\left(\mathrm{M}_{\mathrm{B}}\right)$ and $p$-FMS $\left(\mathrm{M}_{\mathrm{A}}\right)$ with MMA $\left(\mathrm{M}_{\mathrm{B}}\right)$ by $\mathrm{AIBN}$ at $60^{\circ} \mathrm{C}$. $\mathrm{M}_{\mathrm{B}}$ (St): O, experimental; - calculated from $r_{\mathrm{A}}=$ 1.3 and $r_{\mathrm{B}}=0.44$. $\mathrm{M}_{\mathrm{B}}$ (MMA): $\bigcirc$, experimental; - - , calculated from $r_{\mathrm{A}}=0.84$ and $r_{\mathrm{B}}=0.30$.

copolymerization of $p$-FMS with MMA is similar in trend to that of $p$-CMS with MMA.

The copolymerization parameters are listed in Table V. The copolymerization reactivities of $p$-FMS with St or MMA are a little larger than those of $p$-CMS with St or MMA. The AlfreyPrice $Q$ value of $p$-FMS is somewhat larger than that of $p$-CMS. In the case of copolymerization with $\mathrm{St}$, the $e$ value of $p$-FMS is 
R. Asami, M. Gyi, M. TAKaki, and T. IkUta

Table IV. Radical copolymerization of $p$-FMS with MMA ${ }^{a}$

\begin{tabular}{cccccccc}
\hline No. & $\begin{array}{c}p \text {-FMS } \\
\left(\mathbf{M}_{\mathrm{A}}\right) \\
\text { mmol }\end{array}$ & $\begin{array}{c}\text { MMA } \\
\left(\mathbf{M}_{\mathrm{B}}\right) \\
\text { mmol }\end{array}$ & $\begin{array}{c}\text { Mol fract } \\
\text { of } \mathbf{M}_{\mathrm{A}} \\
\text { in feed }\end{array}$ & $\begin{array}{c}\text { Time, } \\
\mathrm{hr}\end{array}$ & $\begin{array}{c}\text { Conversion, } \\
\%\end{array}$ & $M_{n} \mathrm{~b} \times 10^{-5}$ & $\begin{array}{c}\text { Mol fract } \\
\text { of } \mathrm{M}_{\mathrm{A}} \text { in } \\
\text { copolymerization }\end{array}$ \\
\hline $\mathrm{M}-1$ & 2.13 & 16.8 & 0.113 & 1 & 0.15 & 6.5 & 0.21 \\
$\mathrm{M}-2$ & 4.56 & 11.0 & 0.293 & 2 & 4.98 & - & 0.44 \\
$\mathrm{M}-3$ & 9.22 & 6.15 & 0.600 & 2 & 5.72 & 1.4 & 0.64 \\
$\mathrm{M}-4$ & 11.3 & 4.05 & 0.736 & 2 & 7.81 & - & 0.76 \\
$\mathrm{M}-5$ & 14.2 & 1.66 & 0.895 & 2 & 7.93 & 1.4 & 0.89 \\
\hline
\end{tabular}

a Temp, $60^{\circ} \mathrm{C}$; initiator, AIBN (0.5 wt \%); bulk.

b Based on GPC, calibrated with standard polystyrene.

Table V. Copolymerization parameters

\begin{tabular}{lccccccc}
\multicolumn{1}{c}{$\mathrm{M}_{\mathrm{A}}$} & $\mathrm{M}_{\mathrm{B}}$ & $r_{\mathrm{A}}$ & $r_{\mathrm{B}}$ & $r_{\mathrm{A}} r_{\mathrm{B}}$ & $Q$ & $\boldsymbol{e}$ & ref \\
\hline$p$-FMS & $\mathrm{St}$ & 1.3 & 0.44 & 0.59 & 1.3 & -0.07 & This paper \\
CMS & $\mathrm{St}$ & 1.1 & 0.69 & 0.79 & 0.98 & -0.31 & 2 \\
$p$-FMS & MMA & 0.84 & 0.30 & 0.25 & 1.5 & -0.77 & This paper \\
CMS & MMA & 0.76 & 0.37 & 0.28 & 1.3 & -0.73 & 2 \\
\hline
\end{tabular}

larger than of $p$-CMS. This is reasonable when the order of electronegativity of halogens is considered. However, in the case of copolymerization with MMA, the above relation is reversed; moreover, the $e$ value of $p$-FMS is much smaller than that obtained for the copolymerization with St. This suggests an interaction between a fluoromethyl group and a methoxycarbonyl group, since a fluoromethyl group is stabilized by MMA.

\section{REFERENCES}

1. J. Brandrup and E. H. Immergut, "Polymer
Handbook" 2nd Edition, John Wiley \& Sons, New York, N.Y., 1975, p II-344.

2. R. Asami, M. Gyi, M. Takaki, and K. Shinohara, to be published.

3. D. Landini, F. Montanari, and F. Rolla, Synthesis, 428 (1974).

4. C. M. Starks, J. Am. Chem. Soc., 93, 195 (1971).

5. J. Dockx, Synthesis, 441 (1973).

6. J. Bernstein, J.S. Roth, and W. T. Miller, J. Am. Chem. Soc., 70, 2310 (1948).

7. M. Takaki, R. Asami, and S. Kawakami, Polym. Preprints, Jpn., 26, No. 1, 176 (1977).

8. J. Brandrup and E.H. Immergut, "Polymer Handbook" 2nd Edition, John Wiley \& Sons, New York, N.Y., 1975, p II-67. 